\title{
A REVIEW \\ Mycoviruses and their role in biological control of plant diseases
}

\author{
VIJAY KUMAR AND SUNITA CHANDEL
}

\section{SUMMARY}

Mycoviruses are the viruses that infect fungi and prevalent in all major groups of plant pathogenic and edible fungi. The first mycovirus was reported in 1962 from the cultivated mushroom (Agaricus bisporus); the infected mushrooms developed malformed fruiting bodies, grow slowly, mature early and resulting in serious yield losses. Although the majority of known mycoviruses have dsRNA genomes that are packaged in isometric particles, but there are also some reports of DNA mycoviruses. These are transmitted intracellularly during cell division, sporogenesis and cell fusion, but apparently lack an extracellular route for infection. Their natural host ranges are restricted to individuals within the same or closely related vegetative compatibility groups. Mycoviruses causes debilitating diseases of mushrooms; reduce the virulence of their phytopathogenic fungal hosts, production of killer proteins and increase the thermal tolerance of infected host plant. These fungal-virus systems are precious for the development of novel biocontrol strategies. Hypovirulence associated mycoviruses and killer yeast to control plant diseases is emerging as one of the latest biological tool.

Key Words : Mycoviruses, dsRNA, Hypovirulence, Killer yeast

How to cite this article : Kumar, Vijay and Chandel, Sunita (2016). Mycoviruses and their role in biological control of plant diseases. Internat. J. Plant Sci., 11 (2): 375-382, DOI: 10.15740/HAS/IJPS/11.2/375-382.

Article chronicle : Received : 14.05.2016; Accepted : 29.06.2016 